\title{
Attacking the Chor-Rivest Cryptosystem by Improved Lattice Reduction
}

\author{
C.P. SCHNORR and H.H. HöRNER \\ Johann Wolfgang Goethe-Universität Frankfurt \\ Fachbereich Mathematik/Informatik \\ Postfach 111932, D-60054 Frankfurt a.M., Germany
}

\begin{abstract}
We introduce algorithms for lattice basis reduction that are improvements of the famous $\mathrm{L}^{3}$-algorithm. If a random $\mathrm{L}^{3}$-reduced lattice basis $b_{1}, \ldots, b_{n}$ is given such that the vector of reduced GramSchmidt coefficients $\left(\left\{\mu_{i, j}\right\} \quad 1 \leq j<i \leq n\right)$ is uniformly distributed

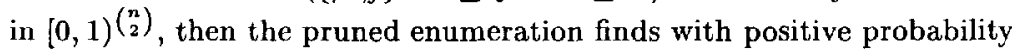
a shortest lattice vector. We demonstrate the power of these algorithms by solving random subset sum problems of arbitrary density with 74 and 82 many weights, by breaking the Chor-Rivest cryptoscheme in dimensions 103 and 151 and by breaking Damgård's hash function.
\end{abstract}

\section{Introduction and Summary}

We address the challenging problem whether it is possible to find, for a given integer lattice basis $b_{1}, \ldots, b_{n} \in \mathbb{Z}^{m}$, in polynomial time a nonzero lattice vector of length $n^{O(1)} \lambda_{1}$, where $\lambda_{1}$ is the minimal length of nonzero lattice vectors. The $\mathrm{L}^{3}$-algorithm of Lenstra,Lenstra, Lovász [LLL82] finds in polynomial time a lattice vector of length $2^{\frac{\pi}{2}} \lambda_{1}$. Schnorr [S87, S94] has extended this algorithm from block size $\beta=2$ to arbitrary block sizes $2 \leq \beta \leq n$. Roughly speaking, this extension goes as follows. Whereas the $\mathrm{L}^{3}$-algorithm iteratively swaps two consecutive basis vectors $b_{i}, b_{i+1}$ if this decreases the length of $\widehat{b}_{i}$, the orthogonal projection of $b_{i}$ in $\operatorname{span}\left(b_{1}, \ldots, b_{i-1}\right)^{\perp}$, block reduction with block size $\beta$ iteratively transforms blocks $b_{i}, b_{i+1}, \ldots, b_{i+\beta-1}$ of $\beta$ consecutive basis vectors as to minimize $\widehat{b}_{i}$. The first vector of a block reduced basis satisfies $\left\|b_{1}\right\| \leq \gamma_{\beta}^{\frac{n-1}{\beta-1}} \lambda_{1}$, where $\gamma_{\beta} \sim \frac{\beta}{\pi e}$ is the Hermite constant of dimension $\beta$. For an implementation of block reduction, see the algorithm BKZ of [SE94]. With block size $\beta=20$ it is only 10 times slower than $\mathrm{L}^{3}$-reduction but for large block sizes $\beta$ the delay factor is about $\beta^{O(\beta)}$. This delay factor is the time to construct a shortest vector $\widehat{b}_{i}$ for a block of size $\beta$ using complete enumeration of all short lattice vectors. A shortest vector of the entire lattice can be found by the algorithm of Kannan [KA87] in exponential time $n^{O(n)}$.

In this paper we present and analyse a new rule for pruning the enumeration of short lattice vectors. This pruning very likely finds a shortest lattice vector, and is exponentially faster than complete enumeration. It is based on the Gaussian volume heuristic that estimates the number of points of lattice $L$ in nice subsets $S \subset \operatorname{span}(L)$ as $\operatorname{vol}(S) / \operatorname{det} L$. If a random $L^{3}$-reduced lattice basis $b_{1}, \ldots, b_{n}$ is given such that the vector of reduced Gram-Schmidt coefficients $\left(\left\{\mu_{i, j}\right\} \quad 1 \leq j<i \leq n\right)$ is uniformly distributed in $[0,1)^{\left(\begin{array}{l}n \\ 2\end{array}\right)}$, then the pruned 
enumeration finds with positive probability a shortest lattice vector. We let $\{r\}$ denote the residue modulo 1 of the real number $r$ in the interval $[0,1)$.

Pruning the enumeration by the Gaussian volume heuristic is more powerful and more flexible than the previous pruning rule of [SE94]. We combine the new pruning with the block reduction algorithm BKZ of [SE94]. 'T'his pruned block reduction is the most powerful lattice reduction algorithm so far. It solves almost all subset sum problems of dimension 74 and 82 for all densities, it breaks the Chor-Rivest cryptosystem in dimensions 103 and 151 , and it easily breaks Damgård's knapsack hash function [DA89]. Our experiments raise new hope that almost shortest lattice vectors can be found in polynomial time.

Lagarias and Odlyzko [LO85] have been the first to solve subset sum problems by lattice reduction. Their attack on subset sum problems of low density was improved by [RK88]. Since then the main progress came from block reduction [SE94], [S87], [S94] and by introducing a superior lattice basis [CJLOSS92]. Kaib and Ritter [KR94] propose an alternative approach based on lattice reduction in the $l_{\infty}-$ norm.

\section{Basic concepts for efficient lattice reduction}

Let $\mathbb{R}^{n}$ be the $m$-dimensional real vector space with ordinary inner product $\langle$,$\rangle and Euclidean length \|y\|=\langle y, y\rangle^{1 / 2}$. A discrete, additive subgroup $L \subset \mathbb{R}^{m}$ is called a lattice. Every lattice is generated by some set of linearly independent vectors $b_{1}, \ldots, b_{n} \in L$, called a basis of $L, L=\left\{t_{1} b_{1}+\cdots+t_{n} b_{n} \mid\right.$ $\left.t_{1}, \ldots, t_{n} \in \mathbb{Z}\right\}$. Let $L\left(b_{1}, \ldots, b_{n}\right)$ denote the lattice with basis $b_{1}, \ldots, b_{n}$. Its rank or dimension is $n$ and its determinant is $\operatorname{det} L=\operatorname{det}\left[\left\langle b_{i}, b_{j}\right\rangle_{1 \leq i, j \leq n}\right]^{1 / 2}$.

With an ordered lattice basis $b_{1}, \ldots, b_{n} \in \mathbb{R}^{m}$ we associate the GramSchmidt orthogonalisation $\widehat{b}_{1}, \ldots, \widehat{b}_{n} \in \mathbb{R}^{m}$ which can be computed together with the Gram-Schmidt coefficients $\mu_{i, j}=\left\langle b_{i}, \widehat{b}_{j}\right\rangle /\left\langle\widehat{b}_{j}, \widehat{b}_{j}\right\rangle$ by the recursion $\widehat{b}_{1}=b_{1}, \quad \widehat{b}_{i}=b_{i}-\sum_{j=1}^{i-1} \mu_{i, j} \widehat{b}_{j} \quad$ for $i=2, \ldots, n$. We let $\pi_{i}$ denote the orthogonal projection $\pi_{i}: \mathbb{R}^{m} \rightarrow \operatorname{span}\left(b_{1}, \ldots, b_{i-1}\right)^{\perp}$ for $i=1, \ldots, n, \quad \pi_{i}\left(b_{j}\right)=$ $\sum_{s=i}^{j} \mu_{s, j} \hat{b}_{s}$. Then $\pi_{i}(L)$ is a lattice of rank $n-i+1$.

An ordered basis $b_{1}, \ldots, b_{n} \in \mathbb{R}^{m}$ is $\mathrm{L}^{3}$-reduced, according to A.K. Lenstra, H.W. Lenstra and L. Lovász [LLL82], with $\delta \in[1 / 4,1)$ if (1) and (2) hold:

$$
\begin{gathered}
\left|\mu_{i, j}\right| \leq 1 / 2 \text { for } 1 \leq j<i \leq n \\
\delta \cdot\left\|\widehat{b}_{k-1}\right\|^{2} \leq\left\|\widehat{b}_{k}+\mu_{k, k-1} \widehat{b}_{k-1}\right\|^{2} \text { for } k=2, \ldots, n .
\end{gathered}
$$

A basis satisfying (1) is called size-reduced. The $\mathrm{L}^{3}$-algorithm of Lovász [LLL82] transforms an integer lattice basis in polynomial time into an $\mathrm{L}^{3}$-reduced basis of the same lattice. Schnorr, Euchner [SE94] propose a floating point version $\mathrm{L}^{3} \mathrm{FP}$ of the $\mathrm{L}^{3}$-algorithm. This algorithm is used whenever we apply $\mathrm{L}^{3}$ reduction.

A lattice basis $b_{1}, \ldots, b_{n}$ is block reduced with block size $\beta$ if it is size reduced and if $\widehat{b}_{i}$, for $i=1, \ldots, n$, is the shortest nonzero vector of the lattice $\pi_{i} L\left(b_{i}, \ldots, b_{\min (i+\beta-1, n)}\right)$. Block reduction has been analysed in [S87], [S94]. 
We consider the following function $c_{t}$ with integer entries $u_{t}, \ldots, u_{n}$

$$
c_{t}\left(u_{t}, \ldots, u_{n}\right):=\left\|\pi_{t} \sum_{i=t}^{n} u_{i} b_{i}\right\|^{2}=\sum_{j=t}^{n}\left(\sum_{i=j}^{n} u_{i} \mu_{i, j}\right)^{2}\left\|\widehat{b}_{j}\right\|^{2} \text { for } t=1, \ldots, n \text {. }
$$

We present the core of the procedure ENUM of [SE94] that generates a shortest lattice vector by complete enumeration in depth first order.

\section{Algorithm ENUM}

INPUT $\left\|\widehat{b}_{i}\right\|^{2}, \mu_{i, t}$ for $1 \leq t \leq i \leq n$.

OUTPUT a minimal nonzero place $\left(u_{1}, \ldots, u_{n}\right)$ and a minimal value $\bar{c}_{1}$ for the function $c_{1}$.

1. FOR $i=1, \ldots, n$ DO $\widetilde{c}_{i}:=u_{i}:=\widetilde{u}_{i}:=y_{i}:=0$

$\widetilde{u}_{1}:=u_{1}:=1, \quad t:=1, \quad \bar{c}_{1}:=\widetilde{c}_{1}:=\left\|\widehat{b}_{1}\right\|^{2}$.

(we always have $\widetilde{c}_{t}=c_{t}\left(\widetilde{u}_{t}, \ldots, \widetilde{u}_{n}\right), \bar{c}_{1}$ is the current minimum of $c_{1}$ )

2. WHILE $t \leq n$

$$
\begin{aligned}
& \widetilde{c}_{t}:=\widetilde{c}_{t+1}+\left(y_{t}+\widetilde{u}_{t}\right)^{2}\left\|\widehat{b}_{t}\right\|^{2} \\
& \text { IF } \widetilde{c}_{t}<\bar{c}_{1} \\
& \text { THEN IF } t>1 \\
& \quad \text { THEN } t:=t-1, \quad y_{t}:=\sum_{i=t+1}^{t_{\text {max }}} \widetilde{u}_{i} \mu_{i, t}, \widetilde{u}_{t}:=\left\lceil-y_{t}\right\rfloor \\
& \quad \text { ELSE } \bar{c}_{1}:=\widetilde{c}_{1}, \quad u_{i}:=\widetilde{u}_{i} \text { for } i=1, \ldots, n
\end{aligned}
$$

ELSE $t:=t+1$

END while

$$
\tilde{u}_{t}:= \begin{cases}\widetilde{u}_{t}+1 & \text { if } t=t_{\max } \\ \operatorname{next}\left(\widetilde{u}_{t},-y_{t}\right) & \text { otherwise }\end{cases}
$$

Here $\lceil r\rfloor \stackrel{\text { def }}{=}\lceil r-0.5\rceil, t_{\text {max }}$ is the maximal previous value of $t$. We define $a^{\prime}=\operatorname{next}(a, r)$ to be the integer which is, next to $a \in \mathbb{Z}$, nearest to $r \in \mathbb{R}$. We have $|a-r| \leq\left|a^{\prime}-r\right| \leq|a-r|+1, \operatorname{sign}\left(a^{\prime}-r\right) \neq \operatorname{sign}(a-r),|a-r|=$ $\left|a^{\prime}-r\right| \Rightarrow a<r<a^{\prime}$.

Correctness. The algorithm ENUM enumerates in depth first order all nonzero integer vectors $\left(\widetilde{u}_{t}, \ldots, \widetilde{u}_{n}\right)$ for $t=1, \ldots, n$ that satisfy $c_{t}\left(\widetilde{u}_{t}, \ldots, \widetilde{u}_{n}\right)$ $\left\langle\bar{c}_{1}\right.$ where $\bar{c}_{1}$ is the actual minimal value for the function $c_{1}$. All enumerated vectors satisfy $\widetilde{u}_{i}>0$ for the largest $i$ with $\widetilde{u}_{i} \neq 0$. For fixed $\widetilde{u}_{t+1}, \ldots, \widetilde{u}_{n}$, the sequence of values $\widetilde{u}_{t}$, generated by iterating the function next $\left(*,-y_{t}\right)$, makes the sequence $c_{t}\left(\widetilde{u}_{t}, \ldots, \widetilde{u}_{n}\right)$ non decreasing. Therefore, if the test $\widetilde{c}_{t}<\bar{c}_{1}$ fails for the current vector $\left(\widetilde{u}_{t}, \ldots, \widetilde{u}_{n}\right)$, the subsequent increment of stage $t$ has the effect to discard all vectors $\left(u, \widetilde{u}_{t+1}, \ldots, \widetilde{u}_{n}\right)$ where $\widetilde{u}_{t}$ preceeds $u$ in the iteration of next $\left(*,-y_{t}\right)$. The discarded vectors can not lead to the minimum of the function $c_{1}$.

\section{Pruning the enumeration}

We prune the enumeration of vectors $\left(\widetilde{u}_{t}, \ldots, \widetilde{u}_{n}\right)$ in ENUM by tightening up the test "IF $\widetilde{c}_{t}<\bar{c}_{1}$ ". We cut off the depth first search at $\left(\widetilde{u}_{t}, \ldots, \widetilde{u}_{n}\right)$ if the 
probability that $\left(\widetilde{u}_{t}, \ldots, \widetilde{u}_{n}\right)$ can be completed as to satisfy $c_{1}\left(\widetilde{u}_{1}, \ldots, \widetilde{u}_{n}\right)<\bar{c}_{1}$ is less than a chosen threshold $2^{-P}$.

The Gaussian volume heuristic. A general principle, dating back to Gauss, estimates the number of points of lattice $L$ in nice subsets $S \subset \operatorname{span}(L)$ as $\operatorname{vol}(S) / \operatorname{det} L$.

How to apply it. Suppose we have chosen integers $\widetilde{u}_{t}, \ldots, \widetilde{u}_{n}$ and we search for $\widetilde{u}_{1}, \ldots, \widetilde{u}_{t-1}$ as to satisfy $c_{1}\left(\widetilde{u}_{1}, \ldots, \widetilde{u}_{n}\right)<\bar{c}_{1}$. We let $\bar{L}$ denote the lattice $\bar{L}=$ $L\left(b_{1}, \ldots, b_{t-1}\right)$. So we want to add to the given lattice vector $b=\sum_{i=t}^{n} \widetilde{u}_{i} b_{i}$ a vector $\vec{b}=\sum_{i=1}^{t-1} \widetilde{u}_{i} b_{i}$ in $\bar{L}$ as to satisfy $\|b+\bar{b}\|^{2}<\bar{c}_{1}$. We decompose $b$ into orthogonal parts $b=y-z$ with $z=-\sum_{j=1}^{t-1} \sum_{i=t}^{n} \widetilde{u}_{i} \mu_{i, j} \widehat{b}_{j} \in \operatorname{span}(\bar{L}), y \in \operatorname{span}(\bar{L})^{\perp}$, $\widetilde{c}_{t}=\|y\|^{2}$. This means, we search for a point in

$$
(b+\bar{L}) \cap S\left(\sqrt{\bar{c}_{1}-\widetilde{c}_{t}}, y\right)=\bar{L} \cap S\left(\sqrt{\bar{c}_{1}-\widetilde{c}_{t}}, z\right)
$$

where $S(r, y)$ is the $(t-1)$-dimensional sphere with radius $r$ and center $y$ in $y+\operatorname{span}(\bar{L})$. Here the equality holds since $z=y-b$. Now we apply the volume heuristic to the lattice $\bar{L}$ and the sphere $S\left(\sqrt{\widetilde{c}_{1}-\widetilde{c}_{t}}, z\right) \subset \operatorname{span}(\bar{L})$. Hence the expected number of vectors $\left(\widetilde{u}_{1}, \ldots, \widetilde{u}_{t-1}\right) \in \mathbb{Z}^{t-1}$ satisfying $c_{1}\left(\widetilde{u}_{1}, \ldots, \widetilde{u}_{n}\right) \leq \bar{c}_{1}$ is $\operatorname{vol} S\left(\sqrt{\bar{c}_{1}-\widetilde{c}_{t}}, z\right) / \operatorname{det} \bar{L}$. We propose to cut off the enumeration of $\left(\widetilde{u}_{1}, \ldots, \widetilde{u}_{t-1}\right)$ if this ratio is less than $2^{-p}$ for a fixed chosen $p$.

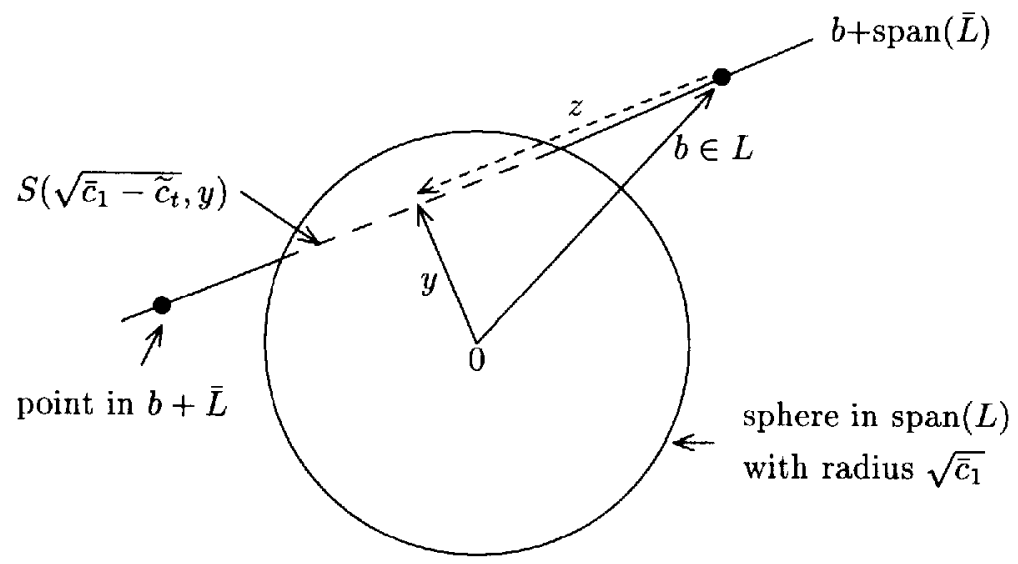

Figure: the volume heuristic 
GAUSS-ENUM. We replace in ENUM the condition "IF $\tilde{c}_{t}<\bar{c}_{1}$ " by "IF vol $S\left(\sqrt{\bar{c}_{1}-\widetilde{c}_{t}}, z\right) / \operatorname{det} \bar{L}<2^{-p}$ ". We call the new procedure GAUSS-ENUM. The parameter $p$ controls the pruning. Large values $p$ correspond to weak pruning, $p=\infty$ corresponds to complete enumeration (no pruning). The inequality $\operatorname{vol} S\left(\sqrt{\bar{c}_{1}-\widetilde{c}_{t}}, z\right) / \operatorname{det} \bar{L}<2^{-p}$ is equivalent to $\widetilde{c}_{t}<\bar{c}_{1}-\eta$ where

$$
\eta=\frac{1}{\pi}\left(\frac{t-1}{2}\right) !^{\frac{2}{t-1}}\left(2^{-p} \prod_{i=1}^{t-1}\left\|\widehat{b}_{i}\right\|\right)^{\frac{2}{t-1}}
$$

If GAUSS-ENUM cuts off the depth first search at $\left(\widetilde{u}_{t}, \ldots, \widetilde{u}_{n}\right)$ the probability, that $\left(\widetilde{u}_{t}, \ldots, \widetilde{u}_{n}\right)$ can be completed as to satisfy $c_{1}\left(\widetilde{u}_{1}, \ldots, \widetilde{u}_{n}\right)<\bar{c}_{1}$, is at most $2^{-p}$. In the analysis of GAUSS ENUM we disregard that GAUSSENUM discards, in addition to the vectors $\left(\widetilde{u}_{1}, \ldots, \widetilde{u}_{n}\right)$, also the vectors $\left(\widetilde{u}_{1}, \ldots, \widetilde{u}_{t-1}, u, \ldots, \widetilde{u}_{n}\right)$ where $\tilde{u}_{t}$ precedes $u$ in the iteration of next $\left(*,-y_{t}\right)$. This can be repaired by a slight change in GAUSS-ENUM. However this yields a reduction algorithm that is less efficient in practice.

Justification of the volume heuristic. The Gaussian principle does not hold in general. MAZO and ODLYZKo [MO90] show that it fails even in the case of spheres and the lattice $L=\mathbb{Z}^{n}$ for particular choices of the center $z$. However the principle holds if the center of the sphere is "uniformly distributed (u.d.) modulo the lattice".

Definition. For a lattice $L$ with basis $b_{1}, \ldots, b_{n}$ a probability distribution of points $\sum_{i=1}^{n} t_{i} b_{i}$ in $\operatorname{span}(L)$ is called u.d. modulo $L$ if the reduced vector $\left(\left\{t_{i}\right\} i=1, \ldots, n\right)$ is u.d. in $[0,1)^{n}$.

This notion does not depend on the choice of the basis. If $b_{1}, \ldots, b_{n}$ and $\bar{b}_{1}, \ldots, \bar{b}_{n}$ are two bases of lattice $L$ there is a matrix $U \in \mathrm{GL}_{n}(\mathbb{Z})$ satisfying $\left[\bar{b}_{1}, \ldots, \bar{b}_{n}\right]=\left[b_{1}, \ldots, b_{n}\right] U$. Since $|\operatorname{det} U|=1$ the linear transformation by $U$ transforms the uniform distribution on $\sum_{i=1}^{n} b_{i}[0,1)$ into the uniform distribution on $\sum_{i=1}^{n} \bar{b}_{i}[0,1)$. Alternatively we can express the uniformity modulo $L$ in terms of the Gram-Schmidt orthogonalization $\widehat{b}_{1}, \ldots, \widehat{b}_{n}$ associated with the basis $b_{1}, \ldots, b_{n}$. The vector $\sum_{i=1}^{n} t_{i}^{\prime} \widehat{b}_{i}$ in $\operatorname{span}(L)$ is u.d. modulo $L$ if and only if the vector $\left(\left\{t_{i}^{t}\right\} i=1, \ldots, n\right)$ is u.d. in $[0,1)^{n}$.

Lemma 1. Let $L$ be a lattice and $S(r, z) \subset \operatorname{span}(L)$ the sphere with fixed radius $r$ and random center $z$ that is u.d. modulo $L$. Then $E_{z} \#(S(r, z) \cap L)=$ $\operatorname{vol} S(r, z) / \operatorname{det} L$ holds for the expectation $E_{z}$.

Proof. For two points $z, \bar{z} \in \operatorname{span}(L)$ that coincide modulo $L$, i.e. $z=$

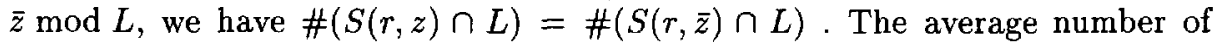
lattice points in $S(r, z)$ is the average number of lattice points per volume part vol $S(r, z)$. Hence the expected value of $\#(S(r, z) \cap L)$ is $\operatorname{vol} S(r, z) / \operatorname{det} L$. 
We apply Lemma 1 to the situation in GAUSS-ENUM with $\widetilde{u}_{t}, \ldots, \widetilde{u}_{n}$ being fixed, $\widetilde{c}_{t}=c_{t}\left(\widetilde{u}_{t}, \ldots, \widetilde{u}_{n}\right), \bar{c}_{1}>\widetilde{c}_{t}$ and a lattice point of $\bar{L}$ is searched in the sphere $S\left(\sqrt{\bar{c}_{1}-\widetilde{c}_{t}}, z\right)$ with center $z=-\sum_{j=1}^{t-1} \sum_{i=t}^{n} \widetilde{u}_{i} \mu_{i, j} \widehat{b}_{j}$.

Theorem 2. If the vector $\left(\left\{\mu_{i, j}\right\} 1 \leq j<i \leq n\right)$ is u.d. in $[0,1)^{\left(\begin{array}{l}n \\ 2\end{array}\right)}$ then for every fixed nonzero $\left(\widetilde{u}_{t}, \ldots, \widetilde{u}_{n}\right) \in \mathbb{Z}^{n-t+1}$ the center $z$ is 1 .d. modulo the lattice $\bar{L}=L\left(b_{1}, \ldots, b_{t-1}\right)$. Moreover

$$
E_{z} \#\left[\left(\widetilde{u}_{1}, \ldots, \widetilde{u}_{t-1}\right) \in \mathbb{Z}^{t-1}: c_{1}\left(\widetilde{u}_{1}, \ldots, \tilde{u}_{n}\right) \leq \bar{c}_{1}\right]=\operatorname{vol} S\left(\sqrt{\bar{c}_{1}-\widetilde{c}_{t}}, z\right) / \operatorname{det} \bar{L} \text {. }
$$

Proof. We can assume that $\widetilde{u}_{n} \neq 0$ since otherwise we can decrease $n$. We see that the vectors $\left(\left\{\widetilde{u}_{n} \mu_{n, j}\right\} j=1, \ldots, t-1\right)$ and $\left(\left\{\sum_{i=t}^{n} \widetilde{u}_{i} \mu_{i, j}\right\} j=1, \ldots, t-1\right)$ are u.d., in $[0,1)^{t-1}$. This shows that $z$ is u.d. modulo $\bar{L}$. Since

$$
\#\left[\left(\widetilde{u}_{1}, \ldots, \widetilde{u}_{t-1}\right) \in \mathbb{Z}^{t-1}: c_{1}\left(\widetilde{u}_{1}, \ldots, \widetilde{u}_{n}\right) \leq \bar{c}_{1}\right]=\#\left(S\left(\sqrt{\bar{c}_{1}-\widetilde{c}_{t}}, z\right) \cap \bar{L}\right)
$$

the expression for the expectation $E_{z}$ follows from Lemma 1.

Success rate of GAUSS-ENUM. Suppose a distribution of $\mathrm{L}^{3}$-reduced lattice bases so that the vector $\left(\left\{\mu_{i, j}\right\} \quad 1 \leq j<i \leq n\right)$ is u.d. in $[0,1)^{\left(\begin{array}{l}n \\ 2\end{array}\right)}$ and let $p>\log _{2} n$. Whenever the depth first search is cut off at a fixed vector $\left(\tilde{u}_{t}, \ldots, \widetilde{u}_{n}\right) \in \mathbb{Z}^{n-t+1}$ then, by theorem 2 , the event that a lattice vector shorter than $\sqrt{\bar{c}_{1}}$ gets lost, has probability at most $2^{-p}$. Therefore the probability of missing the shortest lattice vector is at most $2^{-p}$ times the average number of cutoffs. While the number of cutoffs can be arbitrarily large for badly reduced bases statistical experiments show that, for random $\mathrm{L}^{3}$-reduced basis, the average number of cutoffs is proportional to $c_{p, n} 2^{p}$ where the factor $c_{p, n}$ decreases to 0 as $p$ increases. E.g. for $n<30$ and $p=7$ the probability of success is at least 0.1 .

Expected time bound for GAUSS-ENUM. Using even more heuristic arguments we can show for $p>\log _{2} n$ : Given a random basis $b_{1}, \ldots, b_{n}$ and $\bar{c}_{1} \leq\left\|b_{1}\right\|^{2}$, GAUSS-ENUM performs on the average only $O\left(n^{2} 2^{p}\right)$ arithmetic steps to find a lattice vector $b$ with $\|b\|^{2}<\bar{c}_{1}$, respectively to terminate if such $b$ does not exist.

\section{Solving subset sum problems}

Given positive integers $a_{1}, \ldots, a_{n}, s$ we wish to solve the equation $\sum_{i=1}^{n} a_{i} x_{i}$ $=s$ with $x_{1}, \ldots, x_{n} \in\{0,1\}$. We assume that we are also given $q=\sum_{i=1}^{n} x_{i}$, the number of 1 -entries of the solution. So we search for a $\{0,1\}$-solution $\left(x_{1}, \ldots, x_{n}\right)$ of the two equations $\sum_{i=1}^{n} a_{i} x_{i}=s, \sum_{i=1}^{n} x_{i}=q$. Following [CJLOSS92] we associate to this problem the following lattice basis $b_{0}, \ldots, b_{n} \in \mathbb{Z}^{n+3}$ 


$$
\begin{aligned}
& b_{0}=\left(1, q, q, \ldots q, n^{2} s, n^{2} q\right) \\
& b_{1}=\left(0, n, 0, \ldots 0, n^{2} a_{1}, n^{2}\right) \\
& b_{2}=\left(\begin{array}{ccccc}
0,0, n, \ldots & 0, n^{2} a_{2}, & n^{2}
\end{array}\right) \\
& \vdots=\quad \vdots \quad \vdots \quad \ddots \quad \vdots \quad \vdots \quad \vdots \\
& b_{n}=\left(\begin{array}{ccc}
0,0,0, \ldots & n, n^{2} a_{n}, & n^{2}
\end{array}\right)
\end{aligned}
$$

According to [CJLOSS92] the shortest vector $z$ of the lattice $L\left(b_{0}, \ldots, b_{n}\right)$ solves via (4) almost all subset sum problems of density less then 0.9408 , where the density is $n / \max _{i} \log _{2} a_{i}$. Even beyond this density threshold, solutions of the problems in this paper are associated with very short lattice vectors.

With a $\{0,1\}$-solution $x=\left(x_{1}, \ldots, x_{n}\right)$ of the subset sum problem we associate lattice vectors $z=\left(z_{0}, \ldots, z_{n+2}\right)= \pm\left(-b_{0}+\sum_{i=1}^{n} x_{i} b_{i}\right)$ that satisfy $\left|z_{0}\right|=1, z_{n+1}=z_{n+2}=0, z_{i} / z_{0} \in\{q, q-n\}$ for $i=1, \ldots, n$. Conversely every such lattice vector $z=\left(z_{0}, \ldots, z_{n+2}\right)$ induces a subset sum solution

$$
x_{i}:=\left[\operatorname{IF} z_{i} / z_{0}=q-n \text { THEN } 1 \text { ELSE } 0\right] \text { for } i=1, \ldots, n
$$

We have tested the following algorithm for general subset sum problems with $n=74$ and $n=82$ many weights and for the Chor-Rivest subset sum problem with $n=103$.

\section{Algorithm PRUNED SUBSET SUM}

INPUT lattice basis $b_{0}, \ldots, b_{n} \in \mathbb{Z}^{n+3}$ as in (3).

Perform four successive stages of reduction :

1. $\mathrm{L}^{3}$-reduction.

2. block reduction with block size 20 .

3. pruned block reduction with block size 50 and $p=10$.

4. pruned block reduction with block size 70 and $p=12$.

Algorithmic details. 1. For $\mathrm{L}^{3}$-reduction we use the algorithm $\mathrm{L}^{3} \mathrm{FP}$ of [SE94]. We set $\delta=0.99$, we apply the deep insertion rule of [SE94] for the first basis vector.

2. Block reduction is done by the algorithm BKZ of [SE94] with $\delta=0.99$ resulting in a basis $b_{0}, \ldots, b_{n}$ satisfying for $i=0, \ldots, n$

$$
0.99\left\|\widehat{b}_{i}\right\| \leq\left\|\pi_{i}(b)\right\| \text { for all nonzero } b \in L\left(b_{i}, \ldots, b_{\min (i+\beta-1, n)}\right) .
$$

3. Pruned block reduction is done the same way as block reduction except that we use instead of algorithm ENUM the algorithm GAUSS-ENUM with an appropriate pruning parameter $p$. The resulting basis may occasionally fail the inequalities (5).

4. Test for solution and early termination. Subsequent to every size-reduction of a basis vector $b_{j}$ it is always tested whether $b_{j}$ solves the subset sum problem, i.e. whether (4) induces a solution $x$ for $z=b_{j}$. Also for each stage of the reduction, the vectors of the reduced basis are tested for solution. The algorithm terminates as soon as a solution has been found. 
5. Reduction to the sublattice $\tilde{L}=\left\{\left(z_{0}, \ldots, z_{n+2}\right) \in L\left(b_{0}, \ldots, b_{n}\right): z_{n+1}=\right.$ $\left.z_{n+2}=0\right\}$. After the $\mathrm{L}^{3}$-reduction in stage 1 we construct a basis of the lattice $\tilde{L}$ and we continue the reduction process with this basis. Working with the lattice $\widetilde{L}$ simplifies subsequent reductions since $\operatorname{rank}(\widetilde{L})=\operatorname{rank}(L)-2$. To construct a basis of $\tilde{L}$ we linearly transform the $\mathrm{L}^{3}$-reduced basis $b_{0}, \ldots, b_{n}$ of $L$ so that $b_{i, j}=0$ holds for $i=1, \ldots, n-2$ and $j=n+1, n+2$. Then we eliminate the vectors $b_{n-1}, b_{n}$ from the basis and we remove from the vectors $b_{i} i=0, \ldots, n-2$ the last two coordinates $b_{i, n+1}, b_{i, n+2}$. Upon entry of stage 2 we randomly permute the basis so that it starts with the vectors $b_{i}$ that have a nonzero coordinate $b_{i, 0}$. This enhances the generation of short lattice vectors $z$ which induce via (4) a subset sum solution.

\section{Attacks on the Chor-Rivest cryptosystem}

Chor, Rivest present a public key encryption method for which deciphering has the form of a subset sum problem of high density, for details see [CR88]. Chor, Rivest propose examples of their scheme with $n=197$ and $n=211$ many weights. For testing possible attacks they also designed a small example with $n=103$ many weights and subset sum problems of density 1.271. The Lagarias-Odlyzko method which is based on $\mathrm{L}^{3}$-reduction completely failed for the $n=103$ subset sum problems.

Interestingly, block reduction with pruned enumeration solves the ChorRivest subset sum problems with $n=103$ many weights in only 1.5 hours average time with $42 \%$ success rate. Thus the widespread believe that subset sum problems with density greater than 1 cannot be solved via lattice reduction is outright wrong. The Chor-Rivest scheme with $n=103$ and density 1.271 is even less difficult than random subset sum problems with $n=82$ and density 1 .

Generation of the Chor-Rivest subset sum problems. We take the particular weights $a_{1}, \ldots, a_{103}$ of the example constructed by Chor, Rivest. We generate 50 random vectors $\left(x_{1}, \ldots, x_{103}\right) \in\{0,1\}^{103}$ so that $\sum_{i=1}^{103} x_{i}=12$, and we set $s:=\sum_{i=1}^{103} x_{i} a_{i}$. In the corresponding subset sum problem we are given $a_{1}, \ldots, a_{103}, s$ and have to solve the equations $\sum_{i=1}^{103} x_{i} a_{i}=s, \sum_{i=1}^{103} x_{i}=$ 12 with $x_{1}, \ldots, x_{103} \in\{0,1\}$. (The number 12 arises from the particular construction of the weights $a_{i}$ starting from the field $\mathbb{F}=G F\left(103^{12}\right)$, a generator $g$ for the group of units $\mathbb{F}^{\star}$, an element $t \in \mathbb{F}$ that is algebraic of degree 12 over $G F(103)$, a random permutation $\pi$ in $\operatorname{Sym}(n)$ and a random number $d$ with $0 \leq d<103^{12}-2$, and setting $a_{i}:=\log _{g}(t+\pi(i))+d$ for $i=1, \ldots, 103$.) We solve these 50 subset sum problems by applying the algorithm PRUNED SUBSET SUM to the lattice basis (3) with $n=103, q=12$.

The first table shows, for each of the stages $i=1,2,3,4$, in column 4 the number of successes on stage $i$, in column 5 the number of successes up to stage $i$, in column 6 the average time (with respect to all 50 problems) of stage $i$, in column 7 the total time up to stage $i$ and in column 8 the maximal time of stage $i$. The last column contains the total time for all 50 problems divided by 
the number of successes. All times are in minutes for a HP 715/50 workstation under HP-UX 9.05 .

\begin{tabular}{|c|c|c|c|c|c|c|c|c|}
\hline stage & $\begin{array}{c}\text { block } \\
\text { size }\end{array}$ & $p$ & $\begin{array}{r}\text { \# su } \\
\text { on stage } \\
\end{array}$ & $\begin{array}{l}\text { Iccesses } \\
\text { up to stage }\end{array}$ & tim & $\begin{array}{l}\text { e in mint } \\
\text { av. total }\end{array}$ & $\begin{array}{l}\text { utes } \\
\text { maximal }\end{array}$ & $\mid \begin{array}{c}\text { total time } \\
\text { per success }\end{array}$ \\
\hline 1 & 2 & $\infty$ & 0 & 0 & 0.6 & 0.6 & 0.7 & $\infty$ \\
\hline 2 & 20 & $\infty$ & 3 & 3 & 7.6 & 8.2 & 16.9 & 163.5 \\
\hline 3 & 50 & 10 & 18 & 21 & 86.8 & 95.0 & 247.3 & 226.1 \\
\hline 4 & 70 & 12 & 14 & 35 & 173.4 & 268.4 & 938.3 & 383.5 \\
\hline
\end{tabular}

Stage 1 which performs $\mathrm{L}^{3}$-reduction does not find any solution. This confirms the previous results of Odlyzko showing that $\mathrm{L}^{3}$-reduction is too weak even if the CJLOSS basis (3) is used which is much stronger than the LagariasOdlyzko basis used in the experiments of Odlyzko.

Stage 4 by itself is quite inefficient. It takes a total of 619 minutes per success. This suggests to replace stage 4 by a repetition of stages $1,2,3$ with a randomly permuted input basis. The next table shows the results for two repetitions of stages $1,2,3$.

\begin{tabular}{|c|c|c|c|c|c|}
\hline & $\begin{array}{l}\text { \# succ } \\
\text { in roun }\end{array}$ & $\begin{array}{l}\text { esses } \\
\text { total }\end{array}$ & $\begin{array}{l}\text { time in } \\
\text { average }\end{array}$ & $\begin{array}{l}\text { minutes } \\
\text { av. total }\end{array}$ & $\begin{array}{l}\text { total time } \\
\text { per success }\end{array}$ \\
\hline stages $1,2,3$ & 21 & 21 & 95.0 & 95.0 & 226.1 \\
\hline 1. repetition & 11 & 32 & 65.3 & 160.3 & 250.5 \\
\hline 2. repetition & 6 & 38 & 33.5 & 193.8 & 255.0 \\
\hline
\end{tabular}

With two repetitions the success rate is $76 \%$ with an average time of 3.2 hours. It may be of interest that an alternative algorithm of Ritter, see [KR94], solves all $n=103$ Chor-Rivest problems in about 7 hours maximal time.

Chor-Rivest subset sum problems with more weights. A limited number of first experiments have been carried out by H.H. Hörner in attacking a ChorRivest cryptosystem with $n=151$ many weights and $q=16$ [H94]. So far he could solve 5 out of 50 random problems with an average time of 195 hours for the solved problems.

\section{Attacks on Damgård's knapsack hash function}

In [DA89] a hash function $h$ his proposed based on the subset sum problem. Choose random numbers $a_{1}, \ldots, a_{256}$ in the interval $\left[1,2^{120}-1\right]$ and hash a message $m$ consisting of the bits $m_{1}, \ldots, m_{256}$ into the integer $h\left(m_{1}, \ldots, m_{256}\right)=$ $\sum_{i=1}^{256} a_{i} m_{i}$.

To construct a collision for $h$ it is sufficient to find a nonzero $\{ \pm 1,0\}$-solution $\left(x_{1}, \ldots, x_{256}\right)$ of the equation $\sum_{i=1}^{256} a_{i} x_{i}=0$. This yields messages $m, m^{\prime}$ with bits $m_{i}=\max \left\{0, x_{i}\right\}, m_{i}^{\prime}=-\min \left\{x_{i}, 0\right\}$ for $i=1, \ldots, 256$ satisfying $h(m)=$ $h\left(m^{\prime}\right)$. 
Following an analysis of Joux, Stern [JS94] collisions exist almost surely even for the restricted problem with 80 out of the 256 weights $a_{i}$. We construct nonzero $\{ \pm 1,0\}$-solutions of the equation $\sum_{i=1}^{100} a_{i} x_{i}=0$. We associate to this problem the following lattice basis $b_{1}, \ldots, b_{n} \in \mathbb{Z}^{n+1}$ with $b_{i}=\left(0, \ldots, 1^{(i)}, \ldots, 0, n a_{i}\right)$ for $i=1, \ldots, n$ and $n=100$.

A nonzero lattice vector $z=\left(z_{1}, \ldots, z_{n+1}\right)$ yields a collision if $z_{n+1}=0$ and $\left(z_{1}, \ldots, z_{n}\right) \in\{ \pm 1,0\}^{n}$. We apply to this basis a two-stage reduction consisting of an $\mathrm{L}^{3}$-reduction and a single pruned block reduction with block size 50 and alternative $p$-values $8,9, \ldots, 12$. We test after each size-reduction whether the reduced vector $z$ yields a collision. (The more powerful reduction algorithm PRUNED SUBSET SUM is less efficient since the shortest lattice vector is most likely not in $\{ \pm 1,0\}^{n}$. This follows from the analysis in [JS94].)

Each row in the following table corresponds to 20 random vectors $\left(a_{1}, \ldots\right.$, $\left.a_{100}\right) \in\left[1,2^{120}-1\right)^{100}$. We report the number of successes, the average running time in minutes, the minimal and maximal size of the detected collision ( the size of a collision $\left(x_{1}, \ldots, x_{n}\right) \in\{ \pm 1,0\}^{n}$ is $\left.\#\left\{i: x_{i} \neq 0\right\}\right)$, and the pruning parameter $p$.

\begin{tabular}{|c|r|c|c|c|c|}
\hline block size & $p$ & \# successes & av. time in minutes & min size & max size \\
\hline \hline 50 & 8 & 7 & 235.04 & 48 & 58 \\
\hline 50 & 9 & 16 & 261.98 & 44 & 62 \\
\hline 50 & 10 & 16 & 365.84 & 45 & 59 \\
\hline 50 & 11 & 19 & 388.05 & 37 & 61 \\
\hline 50 & 12 & 20 & 386.65 & 44 & 60 \\
\hline
\end{tabular}

A first collision for Damgård's hash function has been constructed in [JG94] using pruned block reduction via the pruning of [SE94]. They report one success for ten problems. The new results demonstrate the superiority of pruning via the volume heuristic.

\section{General subset sum problems}

We report on solving random subset sum problems of arbitrary density in dimensions $n=74$ and 82 . The previously most powerful algorithm [SE94] could solve almost all problems in dimension $n=66$ by combining block reduction with some sort of pruning. The new algorithm PRUNED SUBSETSUM prunes the enumeration of short lattice vectors by the volume heuristic. It solves for $n=74,82$ a substantial fraction of all random subset sum problems of arbitrary density.

In the following table, every row with entries $n, b$ corresponds to 20 random input bases (3) that are generated as follows. Pick random integers $a_{1}, \ldots, a_{n}$ in the interval $\left[1,2^{b}\right]$, pick a random subset $I \subset\{1, \ldots, n\}$ of size $n / 2$ and put $s=\sum_{i \in I} a_{i}$. To solve the corresponding subset sum problem $\sum_{i=1}^{n} a_{i} x_{i}=s$ we apply the algorithm PRUNED SUBSET SUM to the lattice basis (3) with $q=n / 2$. The numbers in columns $\mathrm{S}, \mathrm{S} 1, \mathrm{~S} 2, \mathrm{~S} 3, \mathrm{~S} 4$ denote the total number of successes, and the number of successes in stages $1,2,3,4$. 


\begin{tabular}{|c|c|c|c|c|c|c|c|c|c|c|c|}
\hline$n$ & $b$ & \multicolumn{5}{|c|}{$\begin{array}{c}\text { \# successes } \\
\mathrm{S}|\mathrm{S} 1| \mathrm{S} 2|\mathrm{~S} 3| \mathrm{S} 4 \\
\end{array}$} & \multicolumn{5}{|c|}{$\begin{array}{l}\text { average time in minutes } \\
1 \mid \text { stage } 2 \mid \text { stage } 3 \mid \text { stage } 4 \mid\end{array}$} \\
\hline 74 & 26 & 20 & 19 & 1 & 0 & 0 & 0.08 & 0.00 & & & 0.08 \\
\hline 74 & 34 & 20 & 5 & 13 & 2 & 0 & 0.13 & 0.23 & 0.05 & & 0.40 \\
\hline 74 & 42 & 19 & 1 & $4 \mid 1$ & 13 & 1 & 0.15 & 1.05 & 0.62 & 0.07 & 1.88 \\
\hline 74 & 50 & 17 & 0 & $\begin{array}{lll}0 & 1\end{array}$ & 11 & 6 & 0.20 & 1.43 & 4.08 & 2.75 & 8.47 \\
\hline 74 & 58 & 15 & 0 & 0 & 4 & 11 & 0.23 & 2.12 & 10.98 & 14.67 & 28.00 \\
\hline 74 & 66 & 12 & 0 & 0 & 6 & 6 & 0.28 & 2.72 & 21.82 & 27.67 & 52.48 \\
\hline 74 & 74 & 12 & 0 & 0 & 6 & 6 & 0.32 & 3.68 & 27.28 & 31.72 & 63.00 \\
\hline 74 & 82 & 20 & 0 & 0 & 19 & 1 & 0.38 & 5:70 & 11.42 & 0.37 & 17.87 \\
\hline 74 & 90 & 20 & 0 & $8 \mid 1$ & 12 & 0 & 0.45 & 4.07 & 2.17 & & 6.68 \\
\hline 74 & 98 & 20 & 0 & 15 & 5 & 0 & 0.55 & 3.00 & 0.38 & & 3.93 \\
\hline 82 & 34 & 20 & \begin{tabular}{l|l}
2 & 1
\end{tabular} & \begin{tabular}{|l|}
16 \\
\end{tabular} & 2 & 0 & 0.17 & 0.37 & 0.05 & & 0.06 \\
\hline 82 & 42 & 20 & 0 & 7 & 13 & 0 & 0.20 & 1.30 & 0.88 & & 2.38 \\
\hline 82 & 50 & 18 & 0 & 1 & 13 & 4 & 0.27 & 1.77 & 4.78 & 1.32 & 8.13 \\
\hline 82 & 58 & 8 & 0 & 0 & 4 & 4 & 0.28 & 2.85 & 10.25 & 26.65 & 40.05 \\
\hline 82 & 66 & 5 & 0 & 0 & 0 & 5 & 0.33 & 3.20 & 30.25 & 65.93 & 99.73 \\
\hline 82 & 74 & 4 & 0 & 0 & 1 & 3 & 0.37 & 3.73 & 60.67 & 171.37 & 236.13 \\
\hline 82 & 82 & 5 & 0 & 0 & 1 & 4 & 0.45 & 4.73 & 104.87 & 172.53 & 282.06 \\
\hline 82 & 90 & 14 & 0 & 0 & 9 & 5 & 0.53 & 6.00 & 60.95 & 73.72 & 141.20 \\
\hline 82 & 98 & 20 & 0 & & 17 & 3 & 0.61 & 7.55 & 33.90 & 7.65 & 49.72 \\
\hline 82 & 106 & 20 & 0 & & 17 & 0 & 0.68 & 9.87 & 9.28 & & 19.83 \\
\hline
\end{tabular}

PRUNED SUBSET SUM is remarkably efficient for densities less than 0.9408 where the shortest lattice vector most likely yields a solution, see lines $n=74, b \geq$ 82 and $n=82, b \geq 90$. This gives new hope that shortest, or near shortest lattice vectors can be found in polynomial time.

Random subset sum problems with $n=82$ and density 1 are harder than the Chor-Rivest scheme with $n=103$ and density 1.271. Here stage 4 of the algorithm PRUNED SUBSET SUM is necessary for the generation of solutions. Only 5 out of 20 problems for $n=82, b=82$ are solved in 282 minutes. The Chor-Rivest problems are easier because the problem solution yields a shortest lattice vector with no further vector being nearly as short.

\section{References}

[CJLOSS92] M.J. Coster, A. Joux, B.A. LaMacchia, A.M. Odlyzko, C.P. Schnorr and J. Stern: Improved Low-Density Subset Sum Algorithms; comput. complexity 2, Birkhäuser-Verlag Basel (1992), 111-128.

[CR88] B. Chor and R.L. Rivest: A knapsack-type public key cryptosystem based on arithmetic in finite fields; IEEE Trans. Inform. Theory, vol IT-34 (1988), 901-909.

[DA89] I. B. Damgård: A Design Principle for Hash Functions; Advances in Cryptology, Proc. Crypto 89, Springer LNCS 435 (1990), 416-427.

[H94] H.H. Hörner: Verbesserte Gitterbasenreduktion; getestet am Chor-Rivest Kryptosystem und an allgemeinen Rucksack-Problemen. Diplomarbeit, Universitä.t Frankfurt (August 1994). 
[JG94] A. Joux and L. Granboulan: A Practical Attack against Knapsack based Hash Functions; Prodeedings EUROCRYPT'94, Springer LNCS (1994).

[JS94] A. Joux and J. Stern: Lattice Reduction: a Toolbox for the Cryptanalyst, TR DGA/CELAR, ENS (1994).

[KA87] R. Kannan: Minkowski's convex body theorem and integer programming; Math. Oper. Res. 12 (1987), 415-440.

[KR94] M. Kaib and H. Ritter: Block Reduction with Respect to Arbitrary Norms; TR U. Frankfurt (1994).

[LO85] J.C. Lagarias and A.M. Odlyzko: Solving low-density subset sum problems; J. Assoc. Comp. Mach. 32(1) (1985), 229-246.

[LLL82] A.K. Lenstra, H.W. Lenstra Jr. and L. Lovász: Factoring polynomials with rational coefficients; Math. Ann. 261 (1982), 515-534.

[MO90] J.E. Mazo and A.M. Odlyzko: Lattice Points in high-dimensional spheres; Monatsh. Math. 110 (1990), 47-61.

[RK88] S. Radziszowski and D. Kreher: Solving subset sum problems with the $\mathrm{L}^{3}$ algorithm; J. Combin. Math. Combin. Comput. 3 (1988), 49-63.

[S87] C.P. Schnorr: A hierarchy of polynomial time lattice basis reduction algorithms; Theoretical Computer Science 53 (1987), 201-224.

[S94] C.P. Schnorr: Block reduced lattice bases and successive minima; Combinatorics, Probability and Computing 3 (1994), 507-522.

[SE94] C.P. Schnorr and M. Euchner: Lattice Basis Reduction: Improved Practical Algorithms and Solving Subset Sum Problems; Mathematical Programming 66 (1994), 181-199. 